\title{
硅负极添加剂对锂离子电池的影响
}

\author{
冯明燕, 田建华, 刘园园, 单忠强 \\ (天津大学 化工学院, 天津 300072)
}

\begin{abstract}
摘 要: 选用乙炔黑(AB)、SuperP、VulcanXC-72 和 BP2000 四种导电剂, 研究其物化性能及含量对硅电极电化学性 能的影响; 探讨了粘合剂种类和用量对硅电极电化学性能的影响。采用场发射扫描电子显微镜对硅电极的形貌进行 表征; 采用恒流充放电测试及循环伏安法对硅电极的电化学性能进行测试。结果表明, 导电剂 SuperP 具有良好的 导电性、适中的比表面积 $\left(75.8 \mathrm{~m}^{2} / \mathrm{g}\right.$ )和颗粒尺寸 $(39.2 \mathrm{~nm})$, 有利于提高硅负极的循环性能及倍率循环性能。采用 $15 \mathrm{wt} \%$ 的导电剂 SuperP 与 $15 \mathrm{wt} \%$ 的粘合剂 CMC 所制备的电极循环 50 次后可逆比容量保持在 $1143.8 \mathrm{mAh} / \mathrm{g}$ 。
\end{abstract}

关＼cjkstart键＼cjkstart词:锂离子电池; 硅负极; 导电剂; 粘合剂

中图分类号: TM911 文献标识码: A

\section{Effect of Silicon Anode Additives on Lithium Ion Batteries}

FENG Ming-Yan, TIAN Jian-Huan, LIU Yuan-Yuan, SHAN Zhong-Qiang

(School of Chemical Engineering and Technology, Tianjin University, Tianjin 300072, China)

\begin{abstract}
Silicon anode of lithium ion batteries was fabricated with different binders and conductive additives (acetylene black, SuperP, VulcanXC-72, BP2000), and the electrochemical performance was investigated in detail. The effect of morphology and addition amount of conductive additives on the electrochemical performance of silicon electrode were investigated. Then, the appropriate type and content of binder in the silicon electrode was optimized. The morphology of silicon electrodes were characterized by scanning electron microscope(SEM). Electrochemical performances of the silicon electrodes were measured by constant current charge-discharge and cyclic voltammetry(CV). The results shows that SuperP has good electrical conductivity, a suitable surface area of $75.8 \mathrm{~m}^{2} / \mathrm{g}$ and average particle size of $39.24 \mathrm{~nm}$, which can improve cycling performance and rate performance of the silicon electrode. With $15 \mathrm{wt} \%$ SuperP and $15 \mathrm{wt} \% \mathrm{CMC}$, the electrode exhibits a reversible capacity of $1143.8 \mathrm{mAh} / \mathrm{g}$ after 50 cycles.
\end{abstract}

Key words: lithium ion batteries; silicon anode; conductive additives; binders

商品化的锂离子电池负极材料主要是石墨碳材 料, 其理论容量仅为 $372 \mathrm{mAh} / \mathrm{g}$, 寻找更高容量和 更为环保的非碳类负极材料是目前锂离子电池负极 材料研究的重要方向之一。硅材料具有最高的理论 比容量 $(4200 \mathrm{mAh} / \mathrm{g})$ 和适中的电位平台 $(\sim 0.4 \mathrm{~V} \mathrm{vs}$ $\left.\mathrm{Li} / \mathrm{Li}^{+}\right)$, 具有独特的优势和发展潜力。并且, 硅是地 壳中分布最多的元素之一，资源丰富，价格低廉，
是具有竞争力的环保类材料 ${ }^{[1-3]}$ 。

然而硅作为锂离子负极材料存在诸多问题。首 先，硅在充放电的过程中会产生剧烈的体积效应 (>300\%), 造成结构塌陷, 从而使循环性能迅速下 降; 另外, 硅的本征导电率低, 仅有 $6.7 \times 10^{-4} \mathrm{~S} / \mathrm{cm}^{[4-6]}$ 。 针对上述问题，研究工作主要集中在合成不同结构 的硅/碳复合材料来改善其导电性与循环性能。譬如,

收稿日期：2014-12-05; 收到修改稿日期：2015-02-27

基金项目：国家自然科学基金(11372217); 天津市重点基金项目(14JCZDJC32400)

National Natural Science Foundation of China (11372217); Tianjin Committee of Science and Technoogy (14JCZDJC32400)

作者简介: 冯明燕(1990-), 女，硕士研究生. E-mail: fmyxinya125@126.com

通讯作者：田建华，教授. E-mail: jhtian@tju.edu.cn 
$\mathrm{Hu}$ 等 ${ }^{[7]}$ 以葡萄糖为碳源, 先进行水热反应然后炭化 得到核壳结构的 $\mathrm{Si} @ \mathrm{SiO}_{x} / \mathrm{C}$ 纳米复合材料, 测得循 环可逆比容量达 $1100 \mathrm{mAh} / \mathrm{g}$, 循环 60 次后比容量无 衰减。Hwa 等 ${ }^{[8]}$ 以 PVA 为碳源, 通过 $750{ }^{\circ} \mathrm{C}$ 高温炭化 得到核壳结构的纳米硅/碳复合材料, 在 $100 \mathrm{~mA} / \mathrm{g}$ 电流密度下, 其可逆比容量为 $1800 \mathrm{mAh} / \mathrm{g}$ 。此外, Zhu 等 ${ }^{[9]}$ 采用石墨烯制备核壳结构的复合材料, 在 $200 \mathrm{~mA} / \mathrm{g}$ 电流密度下循环 80 次后, 容量保持在 $1335 \mathrm{mAh} / \mathrm{g}$ 。上述研究采用硅碳复合结构有效地缓 解硅体积膨胀效应, 并在一定程度上改善了硅基材 料的导电性, 然而所加入的导电剂平均含量仍占电 极材料的 $15 \mathrm{wt} \%$ 以上, 说明导电剂对于提高硅负极 电性能起着重要作用。 Liu 等 ${ }^{[10]}$ 研究了硅颗粒尺寸 与导电剂含量之间的关系，当导电剂含量为 $30 \mathrm{wt} \%$, 硅颗粒尺寸为 $3 \mu \mathrm{m}$ 的电极循环 50 次后, 容量保持 率在 $96 \%$ 以上。Ding 等 ${ }^{[11]}$ 也优化了硅电极中的导电 剂 $\mathrm{CB}$ 的含量, 测得 $40 \mathrm{wt} \% \mathrm{CB}$ 电极的电化学性能优 于 $10 \mathrm{wt} \% \mathrm{CB}$ 电极。

尽管对硅电极导电剂的研究取得了许多成果, 但对不同类型导电剂的综合研究较少, 在选择导电 剂时往往缺少必要的物化性能表征和相应的机理分 析, 而这些内容对构建具有稳定电化学循环性能的 硅电极非常重要。本工作选择 AB、SuperP、VolcanXC72 和 BP2000 四种导电剂, 通过综合性能评估和 机理分析确定与硅匹配最好的导电剂类型。本工作 还对粘合剂的种类及用量对硅电极电化学性能的影 响进行了研究。

\section{1 实验方法}

\section{1 电极制备}

将纳米 $\mathrm{Si}$ 粉 $(99.9 \%$, 徐州捷创新材料科技有 限公司)、粘合剂和导电剂按质量比 70:15:15 的比例 称量, 导电剂分别选乙炔黑 AB(DENKA)、BP2000 (美国 Cabot 公司)、VulcanXC-72(美国 Cabot 公司) 和 SuperP(瑞士 Timical 公司), 粘合剂分别为羧甲基 纤维素钠 $(\mathrm{CMC}) 、$ 海藻酸钠 $(\mathrm{AS}) 、$ 聚偏氟乙烯(PVDF) 和聚四氟乙烯(PTFE 60\%乳液)。将混合均匀的粘稠
度适宜的浆料用刮刀涂覆到铜䇴上，放入真空烘箱 中在 $60^{\circ} \mathrm{C}$ 下烘 $12 \mathrm{~h}$ 左右, 干燥后的电极在 $9 \mathrm{MPa}$ 下压实后备用。

\section{2 电池的组装及电化学性能测试}

将上述电极冲成 $\phi 14 \mathrm{~mm}$ 的圆片作正极, 以 $1 \mathrm{~mol} / \mathrm{L} \mathrm{LiPF}_{6} / \mathrm{EC}: \operatorname{DMC}(V: V=1: 1)($ 天津金牛电源材 料有限公司)为电解液, 金属锂 $(99.9 \%$, 天津中能锂 业)作对电极, 多孔聚丙烯膜 Celgard 2000(美国 Celgard公司)作隔膜, 在 Ar 保护的手套箱(南京大学 仪器厂)中组装 2032 扣式电池。电池在 $0.01 \sim 1.5 \mathrm{~V}$ 之间以电流密度为 $400 \mathrm{~mA} / \mathrm{g}$ (无特殊说明)恒流充放 电循环, 并以不同电流进行倍率充放电循环(武汉 LAND-CT2001A); 在电压范围为 $0.01 \sim 1.5 \mathrm{~V}(\mathrm{vs}$ $\mathrm{Li} / \mathrm{Li}^{+}$), 以 $0.1 \mathrm{mV} / \mathrm{s}$ 进行循环伏安测试(CHI660A, 上海辰华有限公司)。

\section{3 物理性质表征}

采用场发射扫描电子显微镜(SEM, S4800, 日 本日立公司)表征添加不同导电剂的硅电极的形 貌。采用比表面积仪(BET, ASAP/2020, USA)来测 试导电剂的比表面积。将硅粉、粘合剂 PVDF 和 导电剂按质量比 70:15:15 的比例称量、混合形成 均匀的浆料后, 用刮刀涂覆到绝缘的 PET 膜上, 烘干加压后形成致密的薄膜, 利用 RTS-8 型四探 针测试仪(广州四探针科技有限公司)对导电剂的 电阻率及电极电阻率特性进行测试。

\section{2 结果与讨论}

\section{1 不同导电剂的物理特性}

采用乙炔黑 $(\mathrm{AB}) 、$ SuperP、VulcanXC-72 和 BP2000 四种碳黑为硅负极的导电剂, 通过 BET、 Nano measurer 软件及 RTS-8 型四探针测试仪测试 4 种导电剂的比表面积、平均颗粒尺寸及电阻率，如 表 1 所示。从表 1 可以看出, SuperP 具有最低的电 阻率，而 VulcanXC-72 和 BP2000 颗粒尺寸小、比表 面积发达, 且电阻率相对较高。

以 $15 \mathrm{wt} \%$ 羧甲基纤维素钠 $(\mathrm{CMC})$ 为粘合剂, 将 粘合剂、导电剂与硅材料按质量比 70:15:15 混合制

表 1 不同导电剂的比表面积、平均颗粒尺寸、电阻率和电极电阻率

Table 1 Surface area, average particle size, electrical conductivity of different conductive additives and electrical resistivity of electrodes with different conductive additives

\begin{tabular}{cllll}
\hline Samples & AB & SuperP & VulcanXC-72 & BP2000 \\
\hline $\mathrm{N}_{2}$ BET surface area $/\left(\mathrm{m}^{2} \cdot \mathrm{g}^{-1}\right)$ & 54.55 & 75.80 & 212.30 & 890.60 \\
Average particle size $/ \mathrm{nm}$ & 37.61 & 39.24 & 18.96 & 18.26 \\
Electrical resistivity $/(\Omega \cdot \mathrm{cm})$ & 0.3995 & 0.2802 & 0.8620 & 0.7336 \\
Electrical resistivity of silicon electrodes $/(\Omega \cdot \mathrm{cm})$ & 2.0303 & 1.7726 & 5.5808 & 5.3292 \\
\hline
\end{tabular}


备的电极，其分布情况如图 1 所示。硅纳米粒子的 平均粒径为 $80 \mathrm{~nm}$, 图片显示存在几个硅粒子之间 的聚集; 图 1(a)与 1(b)中的 AB 与 SuperP 粒子能够 均匀分散在硅粒子周围, 接触界面清晰; 而图 1(c) 和(d)中的显示 BP2000 与 VulcanXC-72 粒子出现明 显的团聚，没有形成良好的导电网络。

\section{2 硅电极的电化学性能}

图 2 是采用四种不同导电剂制备的硅电极的循 环伏安图, 图中伏安曲线为 5 次循环后的测试结果。 可以看出, 采用不同导电剂的硅电极循环伏安曲线 的氧化还原峰的峰值电流、峰值电位以及峰的形状 都有差异。以 $\mathrm{AB}$ 和 SuperP 为导电剂的硅电极, 其 循环曲线的峰值电流相近, 且明显高于以 BP2000 和 VulcanXC-72 为导电剂的硅电极, 而且两对氧化还原 峰清晰, 尤其是 SuperP 为导电剂的硅电极位于 0.18 0.51 V 的氧化还原峰的峰值电位和峰值电流与 可逆电极的判据更为接近, 说明其导电剂对电极结 构和性能的优化, 使对应的硅电极过程有较好的电 化学可逆性。

图 3 为添加不同导电剂的硅电极首次充放电曲线, 循环性能和倍率性能。从图 3(a)可以看出, 首次放 电曲线在 $0.1 \mathrm{~V}$ 左右呈现一个平坦的长电压平台, 对应的是晶态 $\mathrm{Si}$ 与金属 $\mathrm{Li}$ 反应转化成非晶态 $\mathrm{Li}_{x} \mathrm{Si}$ 的过程; 首次充电曲线中, 在 $0.4 \mathrm{~V}$ 左右电压开始 缓慢增大, 该区域对应于 $\mathrm{Li}_{x} \mathrm{Si}$ 中锂逐步脱出最终形 成无定形硅的过程 ${ }^{[12-13]}$ 。分别以 AB、SuperP、
VulcanXC-72 和 BP2000 为导电剂的硅电极首次充电 比容量分别为 $2950.2 、 2971.3 、 2382.4$ 和 $2608.6 \mathrm{mAh} / \mathrm{g}$, 其首次库伦效率分别为 $77.3 \%$ 、80.5\%、69.5\%和 $72.4 \%$ 。影响首次库伦效率的主要因素是电极表面 固体电解质(SEI)膜的形成以及电导的变化。硅电极 在嵌锂过程中，体积膨胀引起拉应力 ${ }^{[14]}$, 导致表面 产生裂纹，这会使表面的 SEI 膜处在不断被破坏和 生成的过程中, 以致影响电池的库伦效率和循环性 能。如果导电剂粒子与硅粒子形成的电极孔结构合 理, 导电剂粒子可以在一定程度缓解体积膨胀引起 拉应力, 有助于缓解 SEI 膜被破坏的程度, 稳定电 极结构, 而使库伦效率得以提高。这是以 SuperP 为 导电剂的硅电极相比其他三种导电剂具有较高的库 伦效率的原因。

从图 3(b)可以看出, 硅电极充放电循环 50 次之 后, 以 SuperP 为导电剂的硅电极具有相对最高的可 逆容量 $1143.8 \mathrm{mAh} / \mathrm{g}$; 同时 SuperP 电极的库伦效率 迅速达到 92.8\%，而以 $\mathrm{AB} 、$ VulcanXC-72 和 BP2000 为导电剂的硅电极分别为 $88.9 \% 、 72.6 \%$ 和 $81.4 \%$ 。 以 SuperP 为导电剂的硅电极 5 次循环后，可形成较 稳定 SEI 膜, 其库伦效率基本保持在 95\%左右。上 述结果表明以 SuperP 为导电剂的硅电极具有较好 的充放电性能。

图 3(c)为添加不同导电剂的硅电极倍率性能。 在 $100 \mathrm{~mA} / \mathrm{g}$ 电流密度下 10 次循环后, 以 SuperP 为 导电剂的硅电极可逆容量为 $2596.5 \mathrm{mAh} / \mathrm{g}$, 容量保
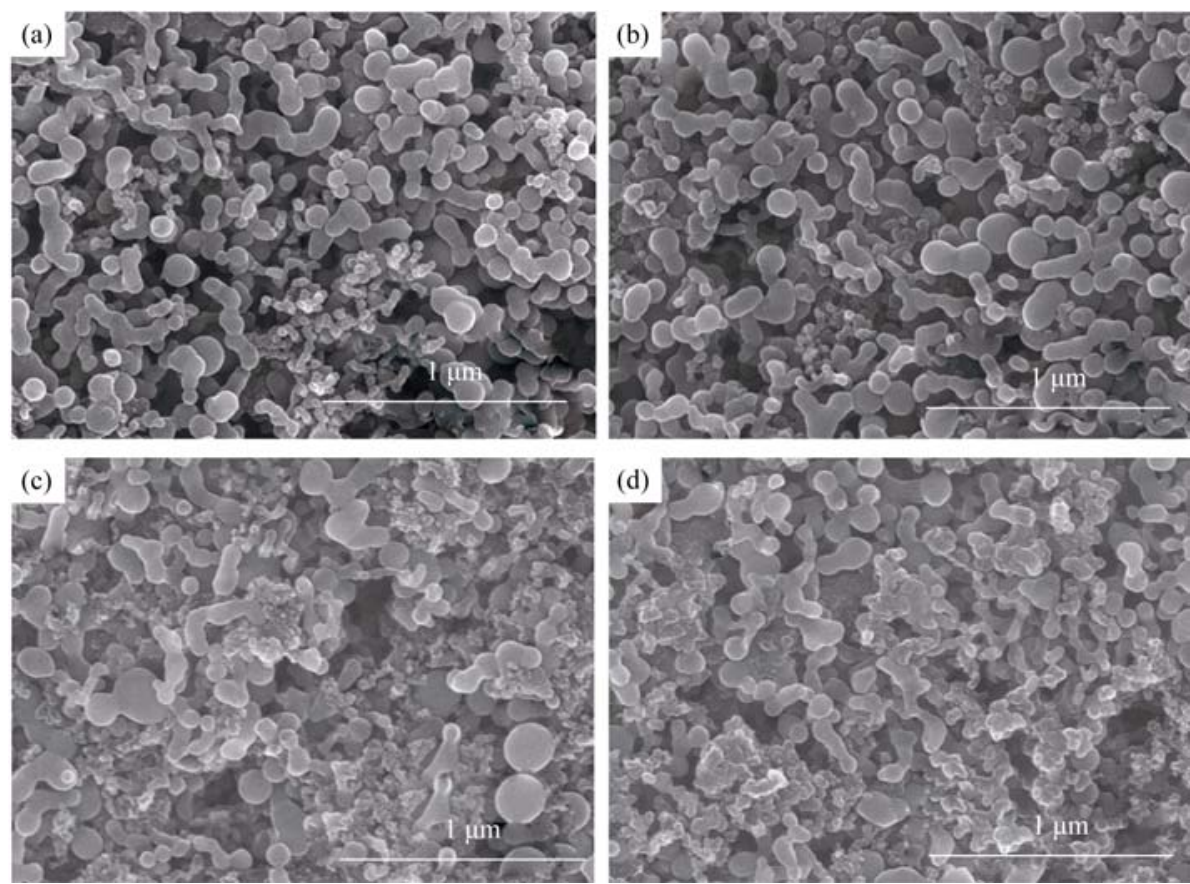

图 1 添加不同导电剂的硅电极的 SEM 照片，粘合剂为 $15 \mathrm{wt} \% \mathrm{CMC}$

Fig. 1 SEM images of silicon electrodes containing $15 \mathrm{wt} \%$ binder CMC with different conductive additives (a) Acetylene black; (b) SuperP; (c) BP2000; (d) VulcanXC-72 


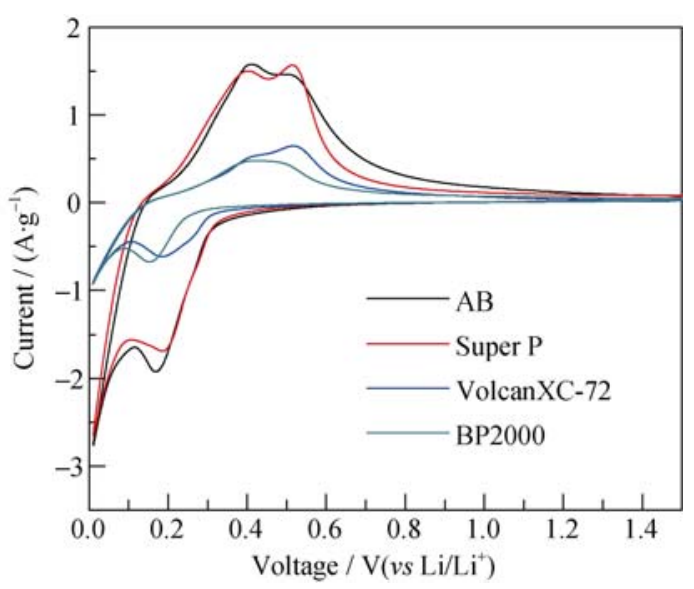

图 2 采用不同导电剂制备的硅电极循环伏安曲线, 粘合剂 为 $15 \mathrm{wt} \% \mathrm{CMC}$

Fig. 2 Cyclic voltammogram curves of silicon electrodes containing $15 \mathrm{wt} \%$ binder $\mathrm{CMC}$ with different conductive additives at potential scanning rate of $0.1 \mathrm{mV} / \mathrm{s}$

持率为 $89 \%$; 当电流增大到 $800 \mathrm{~mA} / \mathrm{g}, 10$ 次循环后, 容量保持在 $1243.4 \mathrm{mAh} / \mathrm{g}$, 是碳类负极理论比容量 $(372 \mathrm{mAh} / \mathrm{g})$ 的 3 倍左右, 而以 $\mathrm{AB} 、$ VulcanXC-72 和 BP2000 为导电剂的硅电极比容量分别是为 567.6、

129.8 和 $399 \mathrm{mAh} / \mathrm{g}$ 。因此, 以 SuperP 为导电剂的硅 电极相比其他三种导电剂具有更好的充放电性能, 电极结构更加的稳定。

\section{3 导电剂的含量对硅电极性能的影响}

导电剂含量较低不利于形成连续的导电网络, 导电剂含量过高时容易因导电粒子的团聚而影响多 孔电极的三维结构而使其有效的电子迁移途径减 少。图 4 是不同 SuperP 加入量硅电极的循环性能, 当 SuperP 加入量为 $15 \mathrm{wt} \%$ 时, 硅电极表现出最好的 电循环性能。对其它三种导电剂的同样实验也得到 了同样的实验结果，这里不再赘述。

\section{4 不同粘合剂及含量对硅电极的影响}

图 5(a)比较了 4 种粘合剂对硅电极循环性能的影 响。以 PVDF 和 PTFE 作为硅材料的粘合剂时, 硅电极 的容量衰减很快, 循环 50 次后衰减到 $100 \mathrm{mAh} / \mathrm{g}$ 左右。 而以 $\mathrm{CMC}$ 和 $\mathrm{AS}$ 作为硅材料的粘合剂时，电池的循环 稳定性得到很大的提高, 循环 50 次后可逆容量分别保 持在 1065.2 和 $1031.4 \mathrm{mAh} / \mathrm{g}$ 。可见 $\mathrm{CMC}$ 和 $\mathrm{AS}$ 有更好 的粘接效果，可以更有效地承受硅材料的体积效应而 提高材料的利用率。这是由于 $\mathrm{CMC}$ 和 $\mathrm{AS}$ 含有的 - $\mathrm{COOH}$ 官能团与硅材料表面的 $\mathrm{SiO}_{2}$ 形成了牢固的氢 键，使导电剂和硅材料更好地粘合在集流体上，从而 稳定电极结构, 提高电池的循环性能 ${ }^{[15-17]}$ 。

鉴于 $\mathrm{CMC}$ 粘合剂对硅负极的电化学性能有所 改善, 为了进一步优化电极的电化学性能, 对 $\mathrm{CMC}$
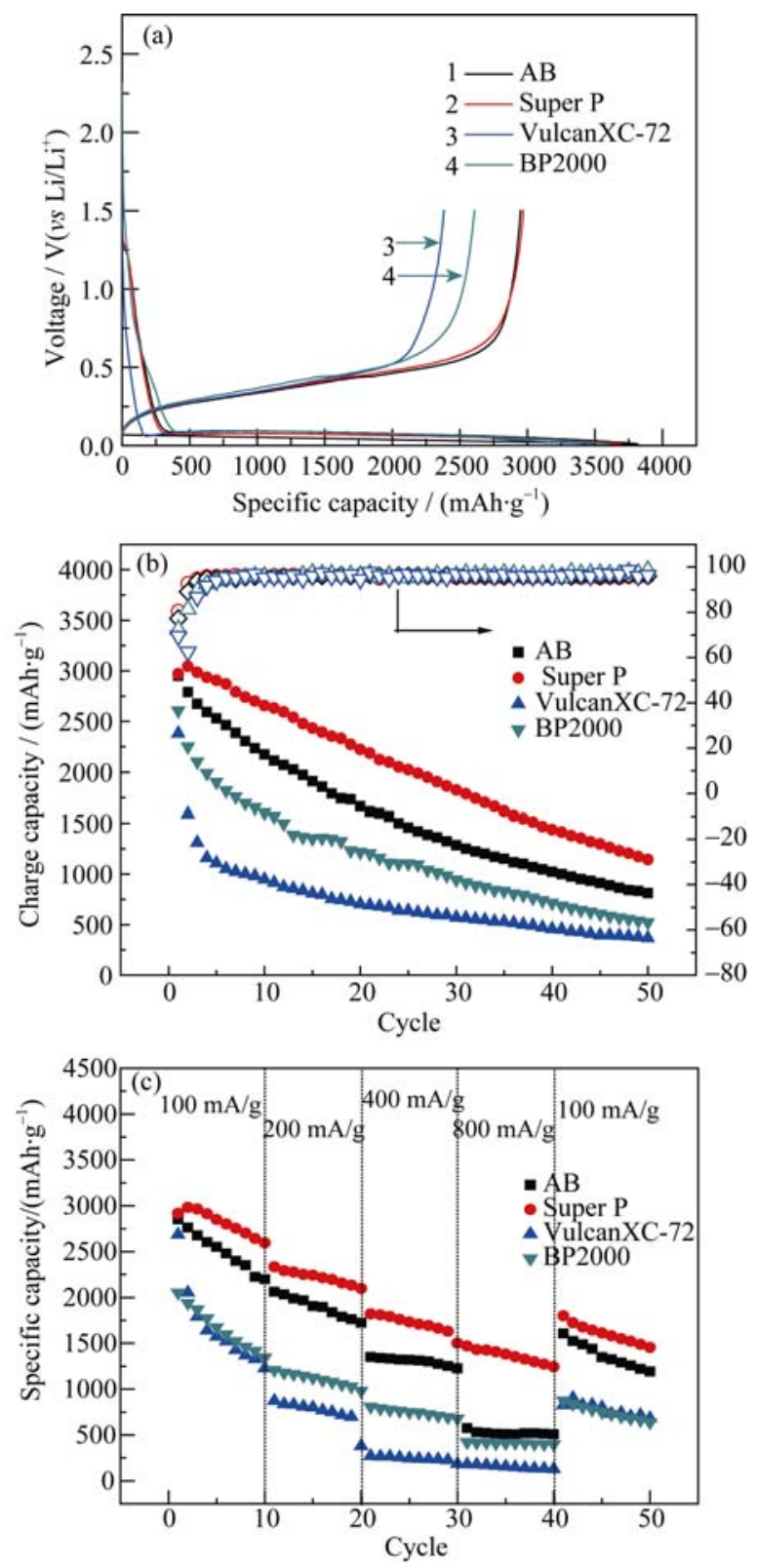

图 3 不同导电剂的硅电极的首次充放电曲线(a), 循环性能 与库伦效率(b)和倍率性能(c), 粘合剂为 $15 \mathrm{wt} \% \mathrm{CMC}$

Fig. 3 First charge-discharge curves (a), cycling performance at $200 \mathrm{~mA} / \mathrm{g}$ (b) and rate performance (c) of silicon electrodes containing $15 \mathrm{wt} \%$ binder $\mathrm{CMC}$ with different conductive additives

粘合剂的用量进行对比分析。图 5(b)比较了 $6 \mathrm{wt} \%$ 、 $10 \mathrm{wt} \% 、 15 \mathrm{wt} \%$ 和 $20 \mathrm{wt} \%$ 粘合剂 CMC 对硅电极循 环性能的影响。当粘合剂的含量过低时, 会造成导 电剂和活性材料与集流体脱落，导致循环性能差; 而过多的粘合剂会增大电极的欧姆电阻，降低电 子转移的速率而影响电池性能。粘合剂含量在 $10 \mathrm{wt} \% \sim 15 \mathrm{wt} \%$ 之间时，随着粘合剂含量的增加， 其电化学性能更加的稳定。

图 6 所示为不同粘合剂制备的硅电极循环 10 次后的 SEM 照片, 从图中可以看出, 当粘合剂为 AS 和 CMC 时, 硅颗粒和导电剂仍可清晰观察到, 


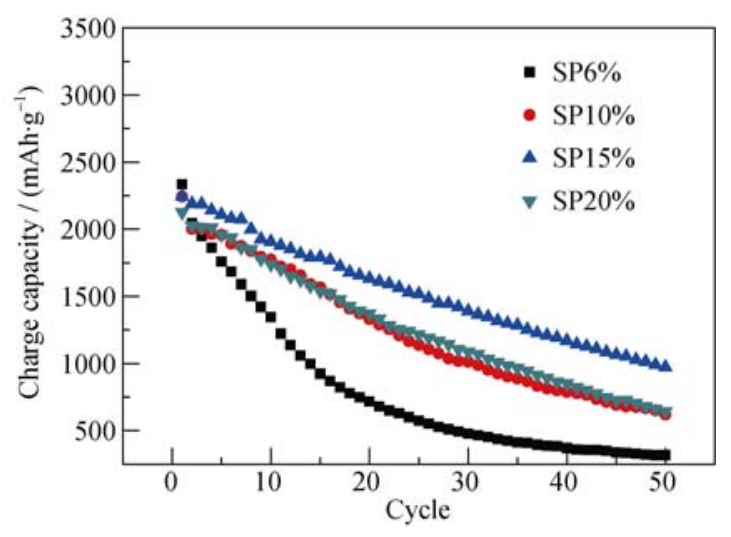

图 4 不同 SuperP 含量的硅电极的循环性能, 粘合剂为 $15 \mathrm{wt} \% \mathrm{CMC}$

Fig. 4 Cycling performance of silicon electrodes containing $15 \mathrm{wt} \%$ binder CMC with different contents of SuperP

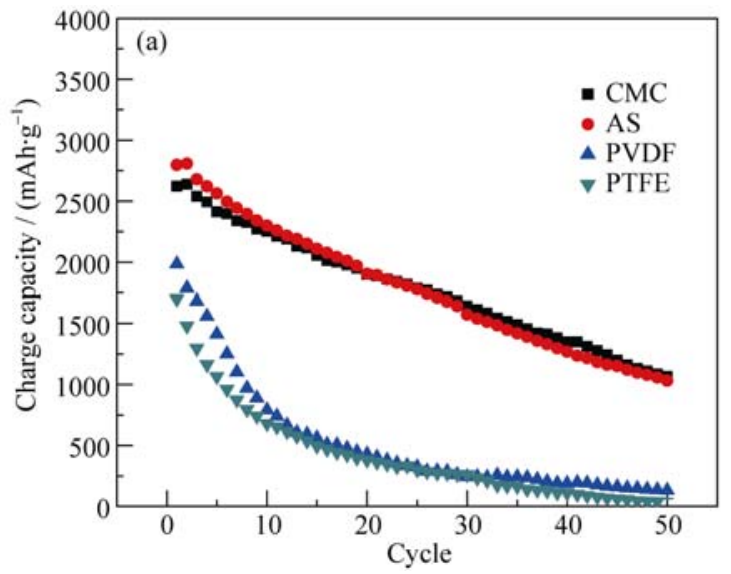

其电极的结构并没有被破坏; 而当粘合剂为 PVDF 和 PTFE 时，硅颗粒因体积膨胀而分裂成小颗粒， 电极的结构也逐渐塌陷。上述结果也证实了粘合剂 AS 和 CMC 有缓冲硅材料的体积膨胀效应和稳定电 极结构的效果。

\section{3 结论}

通过添加不同导电剂来制备硅电极，研究导电 剂的物化性能及其含量对锂离子电池硅负极有着重 要的影响，同时考察了粘合剂的种类及含量对硅电 极的影响。测试结果表明，比表面积大而颗粒尺寸 小的导电剂容易团聚且会加速电解液的分解而影响

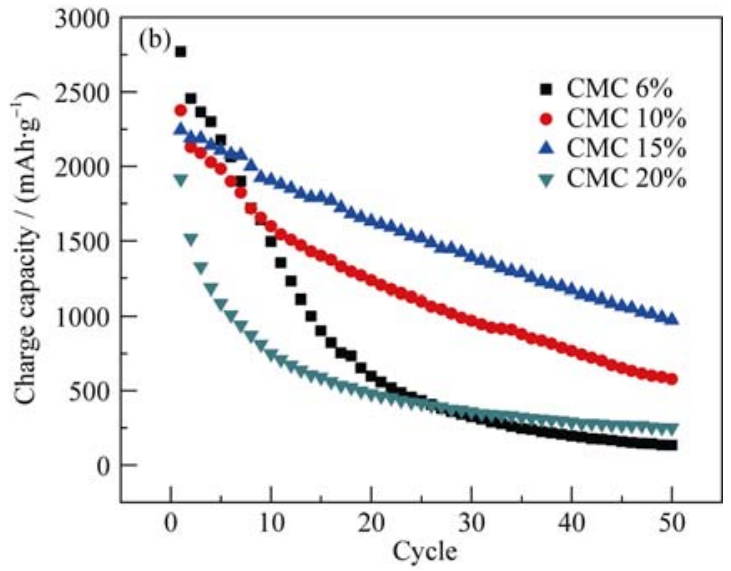

图 5 粘合剂对硅电极的充放电循环性能的影响, 导电剂为 $15 \mathrm{wt} \%$ SuperP

Fig. 5 Influence of binder on the cycle performance of silicon electrodes containing $15 \mathrm{wt} \%$ conductive SuperP (a) Different binders, (b) Different CMC content
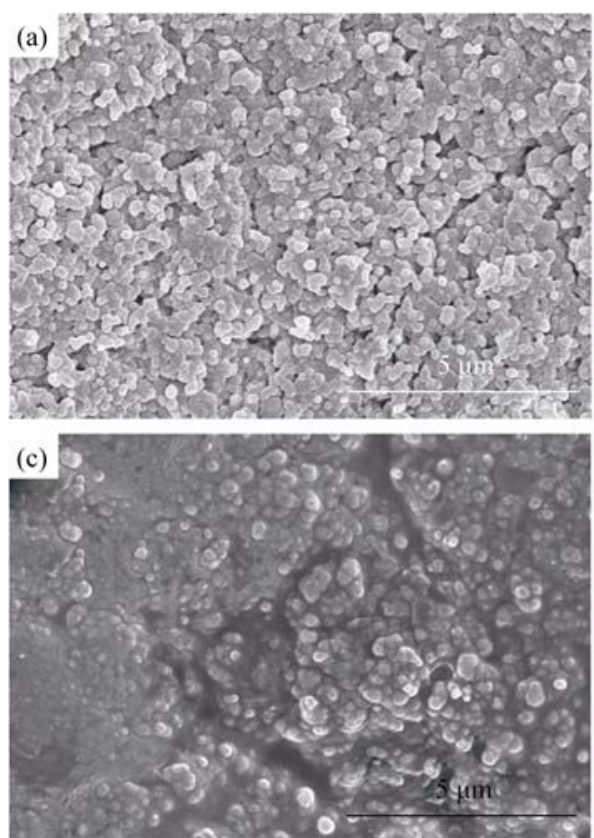
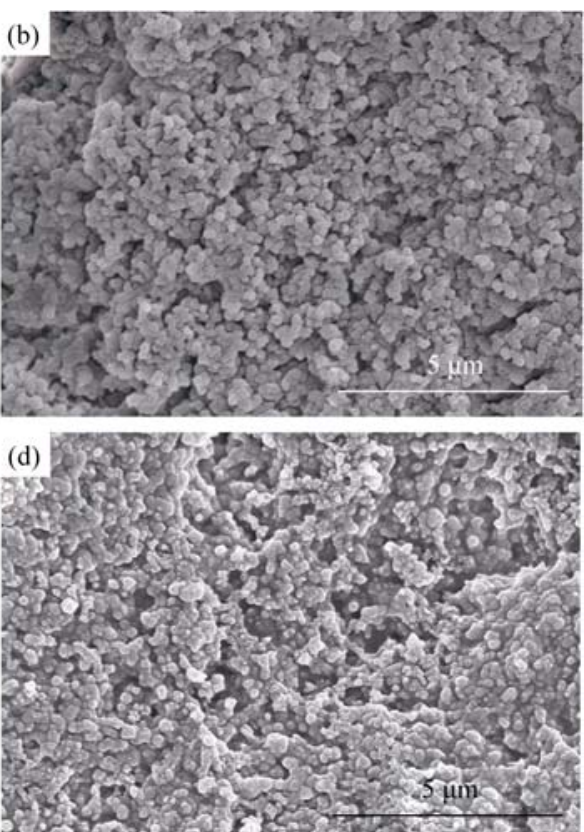

图 6 不同粘合剂制备的硅电极循环 10 次后的 SEM 照片, 导电剂为 $15 \mathrm{wt} \%$ SuperP

Fig. 6 SEM images of silicon electrodes containing $15 \mathrm{wt} \%$ conductive SuperP with different binders after 10 cycles (a) AS; (b) CMC; (c) PVDF; (d) PTFE 
硅电极的电化学性能, 而 SuperP 具有适合的比表面 积 $(75.8 \mathrm{~m} / \mathrm{g})$ 和颗粒尺寸 $(39.2 \mathrm{~nm})$, 且导电性好, 其 制备的硅电极具有较好的循环性能及倍率循环性 能。而以一定量的 CMC 作为粘合剂, 能最大程度减 少硅材料因体积膨胀而造成的损耗, 从而获得结构 稳定的电极。采用 $15 \mathrm{wt} \%$ 的导电剂 SuperP 与 $15 \mathrm{wt}$ $\%$ 的粘合剂 $\mathrm{CMC}$ 制备的硅电极首次充电比容量为 $2971.3 \mathrm{mAh} / \mathrm{g}$, 首次库伦效率为 $80.5 \%$, 循环 50 次 后可逆比容量保持在 $1143.8 \mathrm{mAh} / \mathrm{g}$ 。

\section{参考文献:}

[1] PENG P, LIU Y, WEN Z Y. Electrochemical performance of silicon/carbon/graphite composite anode for lithium ion batteries. $J$. Inorg. Mater., 2013, 28(11): 1195-1199.

[2] KIM H, HAN B, CHOO J, et al. Three-dimensional porous silicon particles for use in high-performance lithium secondary batteries. Angew. Chem., 2008, 120(52): 10305-10308.

[3] KIM H, SEO M, PARK M H, et al. A critical size of silicon nano-anodes for lithium rechargeable batteries. Angew. Chem. Int. Ed, 2010, 49(12): 2146-2149.

[4] SONG T, XIA J, LEE J H, et al. Arrays of sealed silicon nanotubes as anodes for lithium ion batteries. Nano Lett., 2010, 10(5): $1710-1716$.

[5] ZHANG P C, YANG X L, YU D X, et al. Synthesis of silicon/carbon composite anode prepared by in-situ carbothermal reduction for lithium ion batteries. J. Inorg. Chem., 2011, 27(5): 898-902.

[6] XIA F, KIM S B, CHENG H, et al. Facile synthesis of free-standing silicon membranes with three-dimensional nanoarchitecture for anodes of lithium ion batteries. Nano Lett., 2013, 13(7): 3340-3346.

[7] HU Y S, DEMIR C R, TITIRICI M M, et al. Superior storage performance of a $\mathrm{Si} @ \mathrm{SiO}_{x} / \mathrm{C}$ nanocomposite as anode material for lithium-ion batteries. Angew. Chem. Int. Ed, 2008, 47(9): 1645-1649.

[8] HWA Y, KIM W S, HONG S H, et al. High capacity and rate capability of core-shell structured nano-Si/C anode for Li-ion batteries. Electrochimica Acta, 2012, 71: 201-205.

[9] ZHU Y, LIU W, ZHANG X, et al. Directing silicon-graphene self-assembly as a core/shell anode for high-performance lithium-ion batteries. Langmuir, 2013, 29(2): 744-749.

[10] LIU W R, GUO Z Z, YONG W S, et al. Effect of electrode structure on performance of $\mathrm{Si}$ anode in $\mathrm{Li}$-ion batteries: Si particle size and conductive additive. J. Power Sources, 2005, 140(1): 139-144.

[11] DING N, XU J, YAO Y, et al. Improvement of cyclability of Si as anode for Li-ion batteries. J. Power Sources, 2009, 192(2): 644-651.

[12] WANG M S, FAN L Z, HUANG M, et al. Conversion of diatomite to porous $\mathrm{Si} / \mathrm{C}$ composites as promising anode materials for lithiumion batteries. J. Power Sources, 2012, 219: 29-35.

[13] REN J G, WU Q H, HONG G, et al. Silicon-graphene composite anodes for high-energy lithium batteries. Energy Technol., 2013, 1(1): 77-84.

[14] LIU P, SRIDHAR N, ZHANG Y W. Lithiation-induced tensile stress and surface cracking in silicon thin film anode for rechargeable lithium battery. J. Appl. Phys., 2012, 112(9): 093507.

[15] KOVALENKO I, ZDYRKO B, MAGASINSKI A, et al. A major constituent of brown algae for use in high-capacity Li-ion batteries. Science, 2011, 334(6052): 75-79.

[16] KOO B, KIM H, CHO Y, et al. A highly cross-linked polymeric binder for high-performance silicon negative electrodes in lithium ion batteries. Angew. Chem. Int. Ed, 2012, 51(35): $8762-8767$.

[17] YUE L, ZHANG L, ZHONG H. Carboxymethyl chitosan: a new water soluble binder for $\mathrm{Si}$ anode of Li-ion batteries. J. Power Sources, 2014, 247: 327-331. 